\title{
Study on Thickness Effect of Three-Point-Bend Specimen*
}

\author{
Masanori KIKUCHI** and Takehito ISHIHARA ${ }^{* * *}$
}

\begin{abstract}
The thickness effect of a three-point-bend (3PB) specimen on dimple fracture behavior is studied experimentally and numerically. At first, fracture toughness tests were conducted using $3 \mathrm{~PB}$ specimens of different thicknesses. Fracture toughness values and R-curves are obtained, and the thickness effect is discussed. Using scanning electron microscopy (SEM), dimple fracture surfaces are observed precisely. It is found that the thickness effect appears clearly in the void growth process. Finite element (FEM) analyses are conducted based on these experimental data. Using Gurson's constitutive equation, the nucleation and growth of voids during the dimple fracture process are simulated. The distribution patterns of stress triaxiality and the crack growth process are obtained. The results show a good agreement with experimental ones qualitatively. The effects of specimen thickness on R-curves are explained well on the basis of these numerical simulations.
\end{abstract}

Key Words: Constraint Effect, Thickness Effect, Dimple Fracture, $J-R$ Curve, FEM Analysis, Gurson's Constitutive Equation

\section{Introduction}

In elastic-plastic fracture mechanics, the $J$ integral concept $^{(1)}$ is considered to be one of the most important parameters, which controls the fracture process in the crack tip process zone. However, by many experimental studies $^{(2),(3)}$, it has been shown that the apparent $J$ integral value changes due to the change in the constraint condition at the crack tip. This is called the constraint effect. Many studies have been conducted on the effect of the constraint $^{(4),(5)}$. Practically, the local approach method ${ }^{(6),(7)}$ gave one solution to the constraint condition problem. However, the constraint condition on the microscopic fracture process has not been studied well yet. Kikuchi and co-workers have published three papers on the effect of the constraint condition on the fracture toughness of the material. In the first paper ${ }^{(8)}$, the effects of the constraint on the fracture surface and fracture toughness are studied experimentally. In the second paper ${ }^{(9)}$, numerical simulations are conducted using Gurson's constitutive equation. The effect of the constraint condition on the fracture sur-

* Received 11th January, 2006 (No. 04-0403). Japanese Original: Trans. Jpn. Soc. Mech. Eng., Vol.70, No.700, A (2004), pp.1778-1784 (Received 9th April, 2004)

** Department of Mechanical Engineering, Tokyo University of Science, 2641 Yamazaki, Noda-shi, Chiba 278-8510, Japan

*** Graduate School of Tokyo University of Science, 2641 Yamazaki, Noda-shi, Chiba 278-8510, Japan face is discussed. In the third paper ${ }^{(10)}$, similar numerical simulations are conducted, and the effect of the constraint condition on the resistance curve is studied.

Through these studies, the effect of the specimen thickness on the fracture toughness has not been studied well yet. The thickness effect is one of the constraint condition problems and is an important problem in non-linear fracture mechanics. In this study, this problem is studied at first by experiments, and numerical simulations are also conducted. The thickness effect on the microscopic fracture process and fracture toughness are studied and discussed.

\section{Experiments}

Figure 1 shows the shape and size of the three-pointbend specimen (3PB) used in this study. The thickness of the $3 \mathrm{~PB}$ specimen, $B$, is changed in three cases, as $B=2 \mathrm{~mm}, 4 \mathrm{~mm}$ and $8 \mathrm{~mm}$. The material of the specimen is A533B steel, which is used for reactor pressure vessels. The fracture toughness is evaluated on the basis of the JSME testing standard ${ }^{(11)}$. By this standard, the

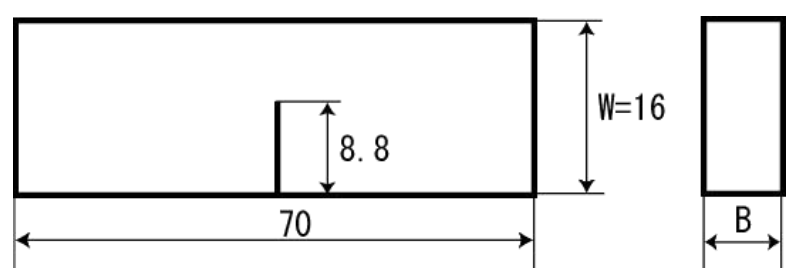

Fig. 1 Three-Point-Bending Specimen (3PB) 


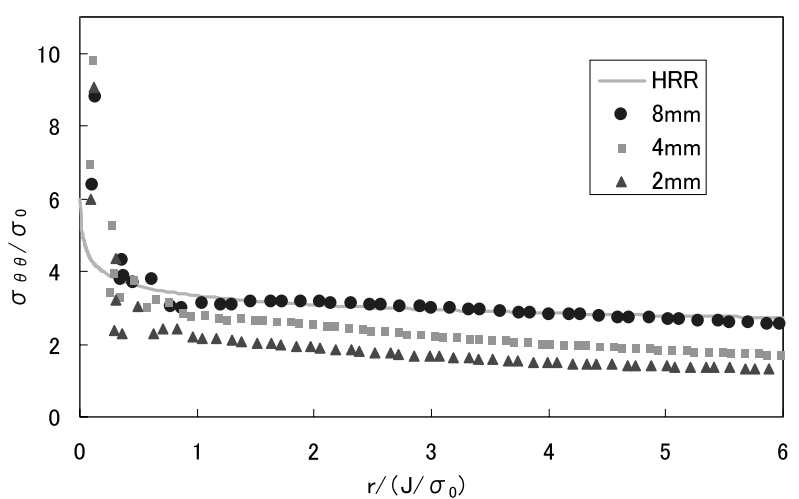

Fig. 2 Crack tip stress fields at center of specimen

Table $1 \quad J_{\text {IC }}$ value of three specimens

\begin{tabular}{|c|c|c|c|}
\hline Thickness $[\mathrm{mm}]$ & 8 & 4 & 2 \\
\hline $\mathrm{J}_{\text {IC }}$ value $[\mathrm{kN} / \mathrm{m}]$ & 158.0 & 194.1 & 171.6 \\
\hline
\end{tabular}

specimen thickness should satisfy the following condition to obtain the valid $J_{\text {IC }}$.

$$
B \geq 25 \frac{J_{\text {in }}}{\sigma_{f s}}
$$

where $J_{i n}$ is $J$ at ductile fracture initiation, and $\sigma_{f s}$ is the flow stress. For this material, these values are $J_{i n}=$ $185 \mathrm{kN} / \mathrm{m}$ and $\sigma_{f s}=660 \mathrm{MPa}^{(12)}$. Using Eq. (1), $B$ should be larger than $7.01 \mathrm{~mm}$. In this study, the 8 -mm-thick specimen satisfies this condition, and the 4-mm and 2mm-thick specimens do not satisfy this condition.

Figure 2 shows the crack tip stress fields for three specimens at the mid-plane of the specimen. They are obtained by three-dimensional elastic-plastic finite element analyses based on $J_{2}$-flow theory. The results are compared with those for the HRR solution ${ }^{(13),(14)}$. For the 8mm-thick specimen, which satisfies Eq. (1), the stress field agrees well with that of the HRR solution. This means that the $J$ integral is a dominant parameter for the crack tip stress field of this specimen. However, for the other 2 specimens, the crack tip stress fields deviate from the HRR field. When the crack tips stress field is lower than the HRR field, it means that the constraint is weak at the crack tip. Figure 2 indicates that the constraint becomes weak as specimen thickness decreases.

In the experiment, the $J$ integral is evaluated using the following conventional equation.

$$
J=\frac{1-v^{2}}{E} K^{2}+\frac{2}{b B} \int_{0}^{\delta} p d \delta
$$

where $K$ is the stress intensity factor, $b$ is the ligament length, $P$ is the load, and $\delta$ is the load-line displacement. The crack growth amount is measured using SEM at 5 points in the (3/8-5/8)B area along the crack front and the average of them is used as $\Delta a$. Table 1 shows the fracture toughness values for each specimen. The fracture toughness of 8-mm-thick specimen is the valid $J_{\mathrm{IC}}$, and the other

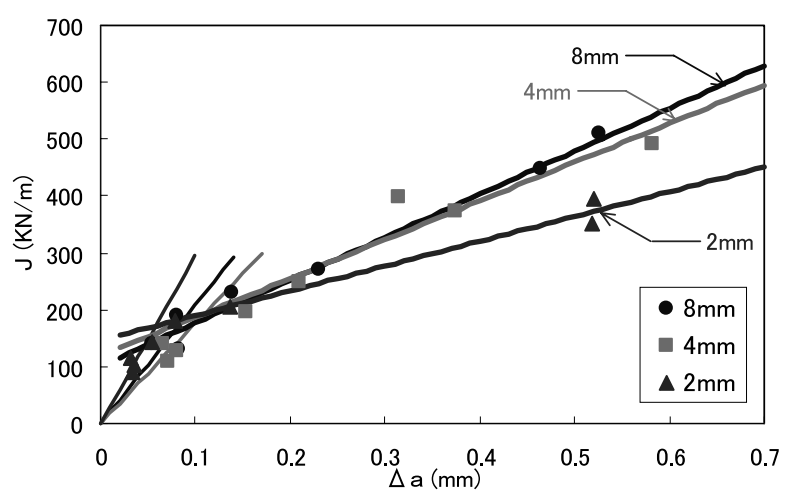

Fig. $3 \quad J-R$ curves obtained by experiments

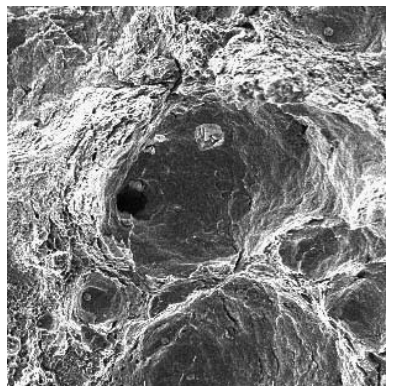

(a) 8-mm-thick specimen

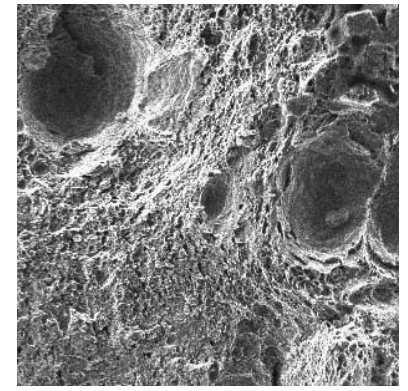

(b) 4-mm-thick specimen

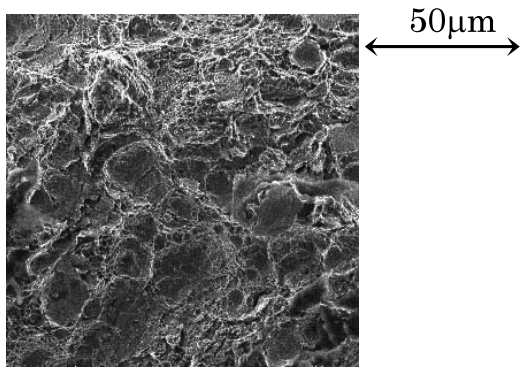

(c) 2-mm-thick specimen

Fig. 4 Fracture surface photographs

two values show are slightly higher than the valid one.

Figure 3 shows $J-R$ curves obtained experimentally. The thickness effect does not appear clearly in blunting lines. However, it appears clearly in resistance curves. As specimen thickness increases, resistance curve increases for a large $\Delta a$ region.

Figure 4 (a)-(c) shows SEM photographs of fracture surfaces for three specimens. They are photographs at the mid-plane of each specimen. Large dimples are observed in the thick specimen, though they are not observed in the thin specimen. In general, the dimple diameter value changes in a wide range. Some voids are larger than $100 \mu \mathrm{m}$, and some are under $1 \mu \mathrm{m}$. In this study, larger voids, which are considered to be nucleated in the early stage of dimple fracture and have a large effect on the fracture process, are mainly considered. The number of voids larger than $10 \mu \mathrm{m}$ is counted for three specimens, and their average diameter is shown in Table 2 . The average dimple 
Table 2 Average of dimple diameter

\begin{tabular}{|c|c|c|c|}
\hline Thickness $[\mathrm{mm}]$ & 8 & 4 & 2 \\
\hline Diameter $[\mu \mathrm{m}]$ & 22.2 & 17.7 & 17.0 \\
\hline
\end{tabular}

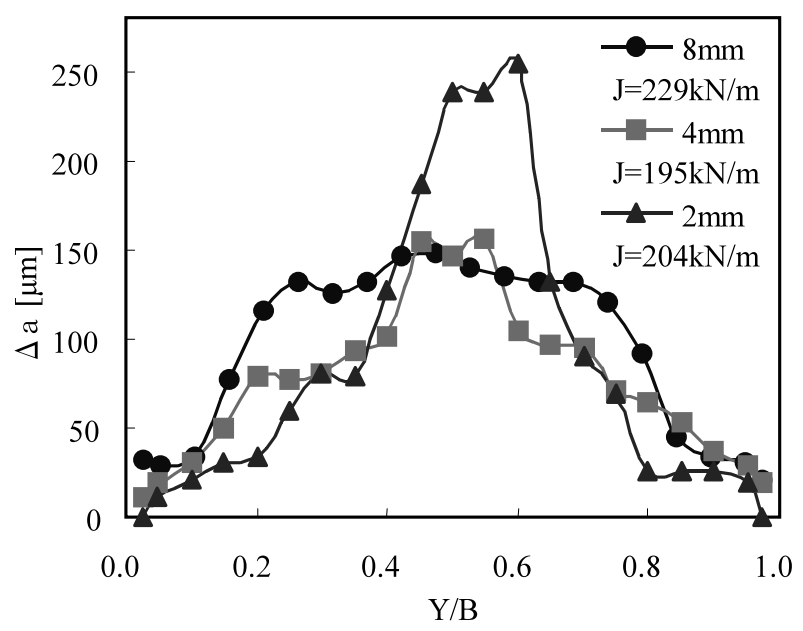

Fig. 5 Crack growth amount along crack tip

diameter changes with specimen thickness. A thick specimen results in a large dimple diameter, and as thickness decreases, dimple diameter also decreases.

Figure 5 shows the crack growth patterns for three specimens. In this figure, the abscissa is the position along the crack front, which is normalized by specimen thickness. Both sides $(Y / B=0.0$ and 1.0) show specimen surfaces, and the center is the mid-plane of the specimen. The ordinate is the crack growth amount. In the 8-mm-thick specimen, the crack growth occurs in a wide area along the crack front. However, in the 2-mm-thick specimen, crack growth occurs mainly in the mid-part of the specimen. As a result, the crack front configuration becomes steep in the thin specimen. In the 2-mm-thick specimen, the fracture mode at the specimen surface is shear-type fracture, and a shear lip is observed.

Through these experiments, it is shown that the constraint condition affects the microscopic fracture processes, such as void nucleation and growth markedly. It is also shown that it has a significant effect on the macroscopic parameter, $J-R$ curve.

\section{FEM Analysis}

\section{1 Gurson's constitutive equation}

To consider the microscopic fracture process, the simulation of the nucleation, growth and coalescence of voids is required. For this purpose, FEM analysis using a constitutive equation proposed by Gurson and later modified by Tvergaard ${ }^{(15)}$ is conducted. This constitutive equation is shown as follows.

$$
\begin{aligned}
\Phi & =\frac{3}{2} \frac{\sigma_{i j}^{\prime} \sigma_{i j}^{\prime}}{\bar{\sigma}_{m}^{2}}+2 f q_{1} \cosh \left(\frac{q_{2} \sigma_{k k}}{2 \bar{\sigma}_{m}}\right)-\left(1+q_{1}^{2} f^{2}\right) \\
& =0
\end{aligned}
$$

Table 3 Material properties (A533B Steel)

\begin{tabular}{|c|c|c|}
\hline Young's modulus & $\mathrm{E}$ & $206[\mathrm{GPa}]$ \\
\hline Poisson's ratio & $v$ & 0.3 \\
\hline Initial yield stress & $\sigma_{\mathrm{ys}}$ & $599[\mathrm{GPa}]$ \\
\hline Critical void volume fraction & $\mathrm{f}_{\mathrm{c}}$ & 0.133 \\
\hline Void volume fraction at final & $\mathrm{f}_{\mathrm{F}}$ & 0.188 \\
\hline Volume fraction of void nucleating & $\mathrm{f}_{\mathrm{N}}$ & 0.02 \\
\hline particles & & \\
\hline Standard deviation of void nucleation & $\mathrm{S}_{\mathrm{N}}$ & 0.1 \\
\hline
\end{tabular}

where $\sigma_{i j}^{\prime}$ is the deviatric stress tensor, $\bar{\sigma}_{m}$ is the equivalent stress, $\overline{\varepsilon_{m}^{p}}$ is the equivalent plastic strain, $f$ is the void volume fraction and $q_{1}$ and $q_{2}$ are constants proposed by Tvergaard. The increment of the void volume fraction, $\dot{f}$, is defined by Eq. (4) as the summation of two terms. One is the growth term, which is defined by Eq. (5) and the other is the nucleation term, defined by Eq. (6).

$$
\begin{aligned}
& \dot{f}=(\dot{f})_{\text {growth }}+(\dot{f})_{\text {nucleation }} \\
& (\dot{f})_{\text {growth }}=(1-f) \dot{\bar{\varepsilon}}_{k k}^{p} \\
& (\dot{f})_{\text {nucleation }}=A\left(\dot{\bar{\sigma}}+\dot{\sigma}_{k k} / 3\right)+B \dot{\bar{\varepsilon}}_{m}^{p}
\end{aligned}
$$

In this paper, plastic strain-controlled nucleation is considered, and $A$ and $B$ in Eq. (6) are given as follows ${ }^{(16)}$.

$$
A=0, B=\frac{f_{N}}{S_{N} \sqrt{2 \pi}} \exp \left[-\frac{1}{2}\left(\frac{\overline{\varepsilon_{m}^{p}}-\varepsilon_{N}}{S_{N}}\right)^{2}\right]
$$

where $f_{N}$ is the volume fraction of void nucleating particles, $S_{N}$ is the standard deviation for probabilistic void nucleation, and $\varepsilon_{N}$ is the mean strain for void nucleation.

\section{2 Numerical model}

As many parameters are required for the use of Gurson's model, they are determined on the basis of the literature $^{(17),(18)}$ as shown in Table 3. It is also pointed out that the numerical results using Gurson's model depend largely on element size. In the following numerical simulation, the minimum element size at the crack tip is determined as $0.025 \mathrm{~mm}$. If we use another mesh size, a slightly different result may be obtained quantitatively. However, in this study, the qualitative evaluation of the thickness effect is the main purpose. Then in the following numerical simulation, the mesh size around the crack tip zone is kept to be the same for every numerical model, and it becomes possible to compare the results of each model and discuss the thickness effect qualitatively. 


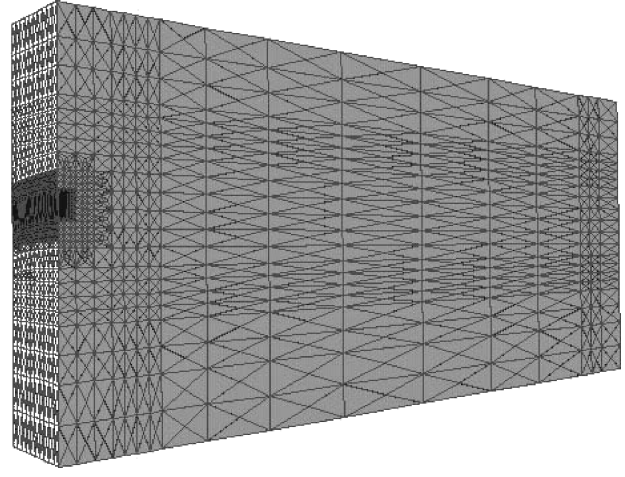

(a) Whole Mesh

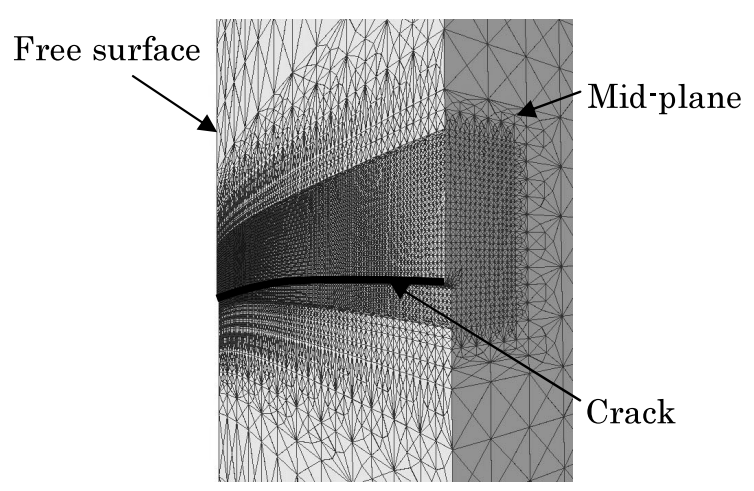

(b) Crack tip

Fig. 6 Mesh pattern of 3PB specimen $(8 \mathrm{~mm})$

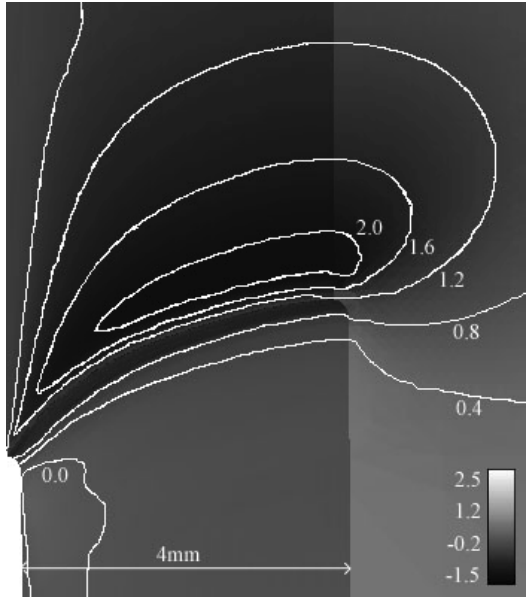

(a) $8 \mathrm{~mm}$ model

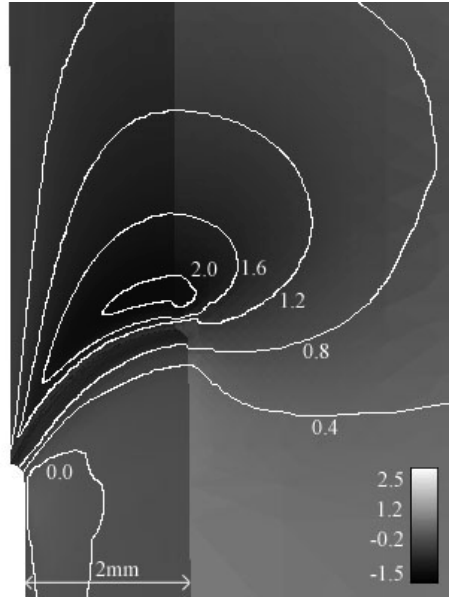

(b) $4 \mathrm{~mm}$ model

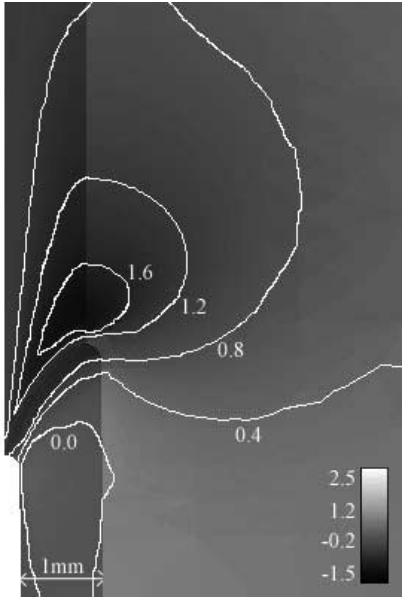

(c) $2 \mathrm{~mm}$ model

Fig. 7 Distribution of stress triaxiality

Table 4 Mesh size

\begin{tabular}{|c|c|c|}
\hline & Nodes & Elements \\
\hline \hline $8 \mathrm{~mm}$ & 474028 & 92900 \\
\hline $4 \mathrm{~mm}$ & 247298 & 48230 \\
\hline $2 \mathrm{~mm}$ & 134568 & 26020 \\
\hline
\end{tabular}

Figure 6 (a) shows the mesh pattern of the 8-mmthick specimen. Figure $6(\mathrm{~b})$ is the mesh pattern around the crack front. A quarter of the specimen is modeled by considering the symmetry of the problem. The right side of this figure shows the mid-plane of the specimen, and the left side is the free surface of the specimen. As the initial crack introduced by fatigue pre-cracking has some curvature, the crack front is modeled by measuring the real crack front configuration obtained experimentally. The total number of elements and the number of nodes for each model are shown in Table 4. As a large number of nodes is used in modeling, a single CPU is not sufficient to solve this problem. A parallel computing technique is employed, and an $8 \mathrm{PC}$ Cluster is used. For the use of Gurson's model, finite deformation analysis is needed.
For this purpose, a super-box element is used for the 3dimensional model in FEM modeling.

\subsection{Thickness effect on fracture surface}

Figure 7 shows the distributions of stress triaxiality around the crack tip for three specimens. Stress triaxiality is defined as the ratio of hydrostatic components to the flow stress. These are results just after the dimple fracture initiation at the mid-plane of the specimens. In Fig. 7(a), which shows the distribution of triaxiality for the 8-mmthick specimen, a high-stress-triaxiality area spreads inside the specimen widely. As the thickness decreases, the high-stress-triaxiality area decreases and the highest value also decreases with it. Stress triaxiality markedly affects the nucleation and growth of voids, which plays a main role in dimple fracture. Figure 8 shows the distributions of stress triaxiality along two lines in front of crack tips. One line is $0.1 \mathrm{~mm}$ and the other one is $0.4 \mathrm{~mm}$ from the crack tip. The abscissa is normalized by the specimen thickness, where $Y / B=0.0$ represents the free surface of the specimen, and $Y / B=0.5$ represents the mid-plane of the specimen. Along both lines, stress triaxiality decreases 
with the decrease in specimen thickness. It is also shown that the high-stress-triaxiality area widely spreads in the thick specimen, and it becomes narrow for the thin specimen. By these figures, it is shown that large voids may be nucleated in a wide area in the thick specimen. As a result, dimple fracture occurs by coalescence of these large voids, and large dimple patterns are observed on the fracture surface in the thick specimen. In the thin specimen, the dimple fracture may occur by the coalescence of smaller voids. These results correspond to the experimental results shown in Fig. 4 and Table 2, where large voids are observed in the thick specimen and voids become small in the thin specimen.

Figure 9 shows the definition of crack growth amount, $\Delta a$, which is used in the following discussion. In the numerical model, the initial curvature at the crack tip is assumed to simulate crack tip blunting. The crack growth amount is defined as the distance between the bottom of this curvature and the edge of the fracture area, as shown in this figure.

Figure 10(a)-(c) shows crack growth patterns for

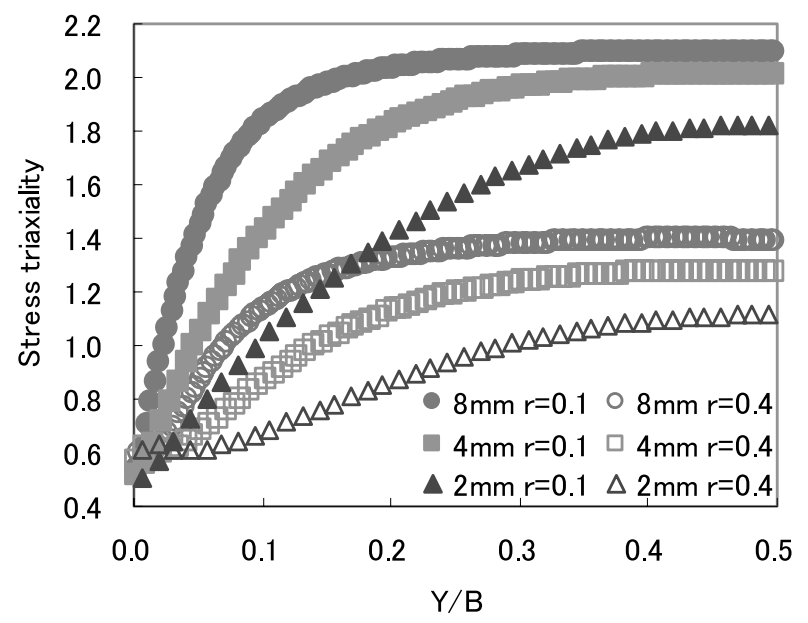

Fig. 8 Stress triaxiality along crack tip three specimens. These figures are the results when $J$ is nearly $200 \mathrm{kN} / \mathrm{m}$, after a large amount of dimple fracture growth. In these figures, the white zones ahead of the initial crack front denote the dimple fracture areas. Dimple fracture occurs in a wide area in the 8-mm-thick specimen. However, in the 2-mm-thick specimen, dimple fracture occurs mainly at the mid-plane, and a steep crack front configuration is generated. Figure 11 shows fracture patterns along the crack front of the three specimens when $J$ is nearly $200 \mathrm{kN} / \mathrm{m}$. In the thick specimen, crack growth amount is nearly constant in the mid-part of the specimen, but it changes markedly in the thin specimen. These fracture patterns are similar to the results of experimental results as shown in Fig. 5.

\section{4 Thickness effect on $\boldsymbol{J}-\boldsymbol{R}$ curves}

Figure 12 shows $J-R$ curves obtained by numerical simulation. The ordinate, $J$ integral value, is evaluated using conventional equation using the load-displacement data obtained numerically. The abscissa represents the crack growth amount. It is determined as the average value measured at 5 points along the crack front, due to the fracture toughness testing standard. Figure 13 shows

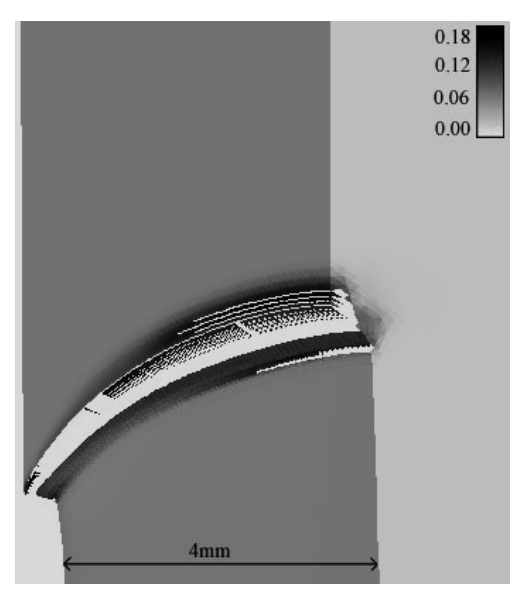

(a) $8 \mathrm{~mm}$ model

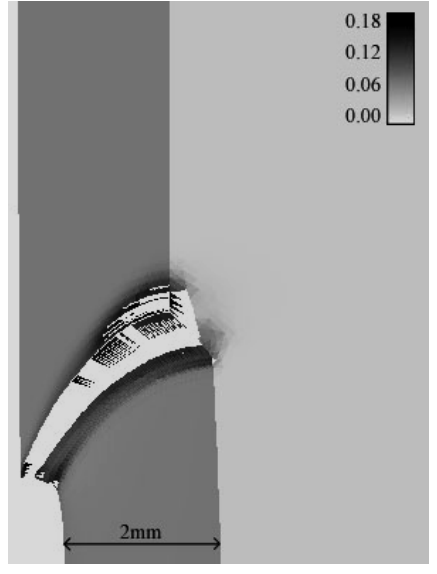

(b) $4 \mathrm{~mm}$ model

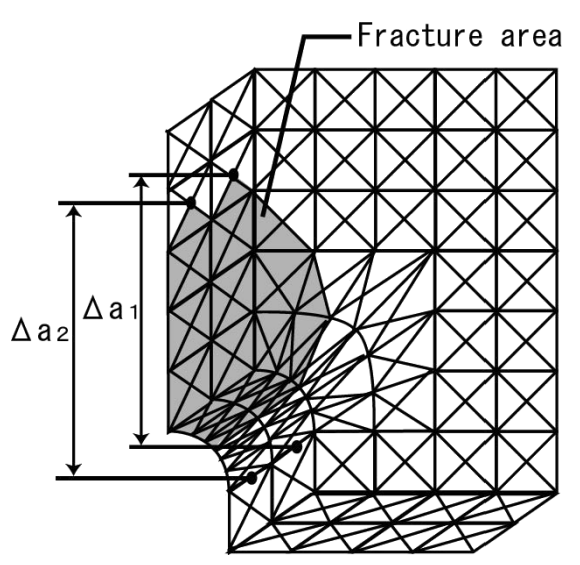

Fig. 9 Definition of crack growth amount

Fig. 10 Distribution of void volume fraction 


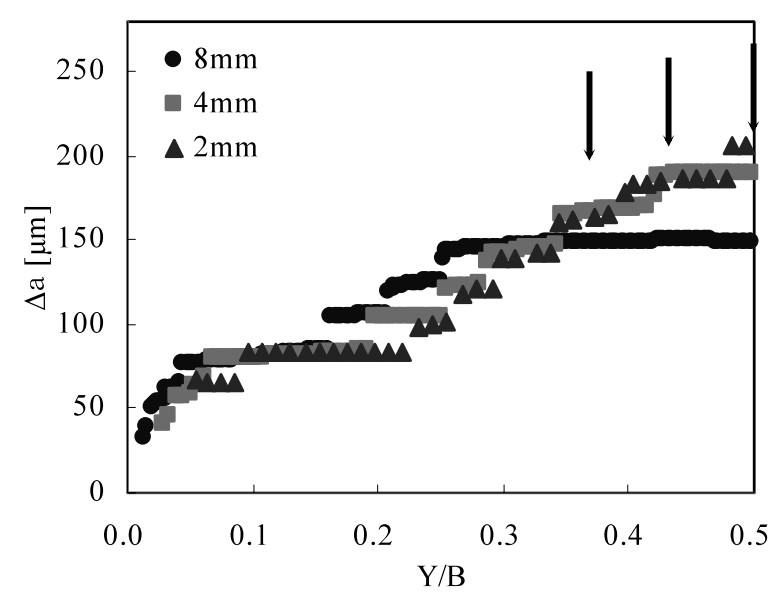

Fig. 11 Fracture pattern along crack tip

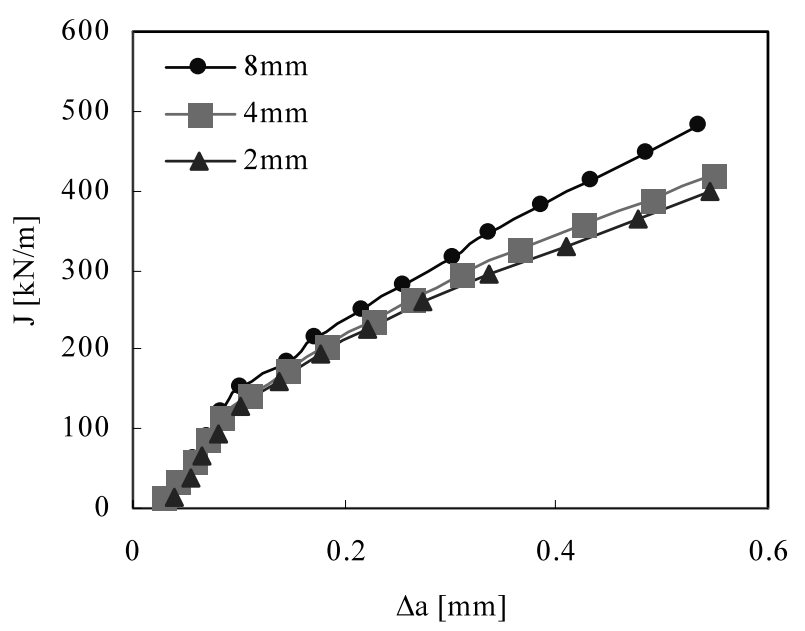

Fig. $12 J-R$ curves obtained by analysis

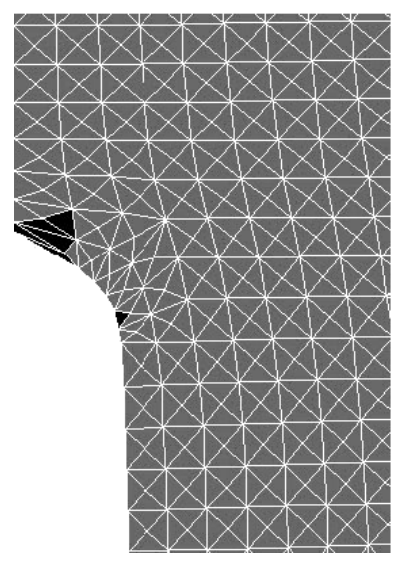

(a) $\Delta a=0.1 \mathrm{~mm}$

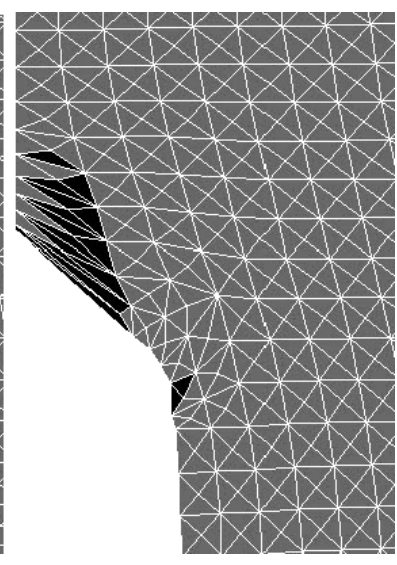

(b) $\Delta a=0.2 \mathrm{~mm}$
Fig. 13 Fracture pattern at center of 8-mm-thick specimen

the fracture pattern at the mid-plane of the specimen for the 8-mm-thick specimen. At the beginning of dimple fracture, $\Delta a=0.1 \mathrm{~mm}$, dimple fracture mainly occurs in elements around the initial curvature of the crack tip. It seems to grow almost perpendicular to the initial crack line. This phenomenon is related to the blunting of the crack tip. The relationship between $J$ and $\Delta a$ in this fracture process is shown by the initial straight lines in Fig. 12. After some amount of crack growth, the fracture grows in the direction parallel to the initial crack line, as shown in Fig. 13 (b). This process corresponds to the resistance curve in Fig. 12. The resistance curve increases as specimen thickness increases as shown in Fig. 12. This result agrees qualitatively with the experimental ones shown in Fig. 3.

\section{Discussion}

In Kikuchi and coworkers' previous papers ${ }^{(8)-(10)}$ the $J-R$ curve becomes high under a low-constraint condition, and a low $J-R$ curve is obtained under a high-constraint condition. In this study, the thick specimen is under a high-constraint condition and the thin specimen is under a low-constraint condition. However, numerical results show that a high-constraint specimen results in a high $J-R$ curve as shown in Fig. 12. This is contrary to the results of previous papers.

The reason for this tendency is the definition of the crack growth amount in Fig. 12. By the fracture toughness standard, the crack growth amount is defined as the average of the measured values at 5 points along the crack front. These 5 points are near the midplane of the specimen. In Fig. 11, the locations of 3 points to measure the crack growth amount on the half thickness of the specimen are shown by arrows. It is obvious that the distribution patterns of crack growth amount at these locations are markedly different in the three specimens. In the thick specimen, crack growth amount is nearly constant along the crack front, but it changes markedly in the thin specimen. At these 3 points, the $\Delta a$ values of the 2 -mm-thick specimen are larger than those of the 8-mm-thick specimen. Then the average crack growth amount of the thin specimen becomes larger than that of the thick specimen. In Table 5, $\Delta a$ values measured by this method when $J$ is almost $200 \mathrm{kN} / \mathrm{m}$ are shown. Both experimental and numerical results are shown in this table. The tendencies of both results are very similar to each other. Table 6 shows the average $\Delta a$ values on the whole specimen thickness for three specimens. In this table, significant differences between each specimen are not recognized. The reason that the low-constraint-condition specimen results in a high resistance is well explained by these figures and tables.

In the real specimen, shear lip fracture occurs near the free surfaces of the specimen. For the thin specimen, the rate of shear lip fracture area with respect to dimple fracture area increases. To fully understand the constraint effect due to specimen thickness, this phenomenon should be studied with the study of dimple fracture inside the specimen, which is our target in the future. 
Table 5 Crack growth amount ( $J_{\text {IC }}$ test $)$

\begin{tabular}{|c|c|c|}
\hline & \multicolumn{2}{|c|}{$\Delta \mathrm{a}[\mu \mathrm{m}](\mathrm{J} \doteqdot 200 \mathrm{kN} / \mathrm{m})$} \\
\hline Thickness & Experiment & Analysis \\
\hline $8 \mathrm{~mm}$ & 138.3 & 149.6 \\
\hline $4 \mathrm{~mm}$ & 148.0 & 182.4 \\
\hline $2 \mathrm{~mm}$ & 192.0 & 190.3 \\
\hline
\end{tabular}

Table 6 Crack growth amount (Average of thickness)

\begin{tabular}{|c|c|c|}
\hline & \multicolumn{2}{|c|}{$\Delta \mathrm{a}[\mu \mathrm{m}](\mathrm{J} \doteqdot 200 \mathrm{kN} / \mathrm{m})$} \\
\hline Thickness & Experiment & Analysis \\
\hline $8 \mathrm{~mm}$ & 92.6 & 119.3 \\
\hline $4 \mathrm{~mm}$ & 74.8 & 124.4 \\
\hline $2 \mathrm{~mm}$ & 83.3 & 125.6 \\
\hline
\end{tabular}

\section{Concluding Remarks}

Thickness effect, which is one of the constraint condition problems, is studied by experiments and also by numerical simulations. It is found that the thickness effect appears in both microscopic and macroscopic dimple fracture processes. The following results are obtained.

(1) Under a low-constraint condition, it is shown that small voids are nucleated and dimple fracture occurs mainly at the mid-plane of the specimen.

(2) It is shown that specimen under a high-constraint results in a low resistance in the $J-R$ curve. This thickness effect is well explained by numerical simulation.

\section{References}

( 1 ) Rice, J.R., A Path Independent Integral and the Approximate Analysis of Strain Concentration by Notches and Cracks, Trans. ASME, J. Applied Mechanics, Ser. E, Vol.90 (1968), pp.379-386.

( 2 ) Sorem, W.A., Dodds, R.H. and Rolfe, S.T., Effects of Crack Depth on Elastic-Plastic Fracture Toughness, Int. J. Fracture, Vol.47 (1991), pp.105-126.

( 3 ) O'Dowd, N.P. and Shih, C.F., Family of CrackTip Fields Characterized by a Triaxiality ParameterI. Structure of Fields, J. Mech. Phys. Solids, Vol.39 (1991), pp.989-1015.

( 4 ) Anderson, T.L., Crack Tip Parameters for Large Scale Yielding and Low Constraint Configurations, Int. J. Fracture, Vol.41 (1989), pp.79-104.
( 5 ) Dodds, R.H., Anderson, T.L. and Kirk, M.T., A Framework to Correlate a/W Ratio Effects on Elastic-Plastic Fracture Toughness (Jc), Int. J. Fracture, Vol.48 (1991), pp.1-22.

( 6 ) Beremin, F.M., A Local Criterion for Cleavage Fracture of a Nuclear Pressure Vessel Steel, Metallurgical Transactions, Vol.14A (1983), pp.2277-2287.

( 7 ) Xia, L. and Shih, C.F., Ductile Crack Growth-III. Transition to Cleavage Fracture Incorporating Statistics, J. Mech. Phys. Solids, Vol.44 (1996), pp.603-639.

( 8 ) Kikuchi, M. and Nagai, M., Constraint Effect at the Crack Tip (2nd Report: Constraint Effect of High Toughness Material), Trans. Jpn. Soc. Mech. Eng., (in Japanese), Vol.64, No.625, A (1998), pp.2367-2373.

( 9 ) Kikuchi, M. and Takahashi, A., Dimple Fracture Simulation under Different Loading Conditions, Trans. Jpn. Soc. Mech. Eng., (in Japanese), Vol.67, No.656, A (2001), pp.665-671.

(10) Kikuchi, M. and Sasaki, J., Dimple Fracture Simulation under the Different Constraint Condition, Trans. Jpn. Soc. Mech. Eng., (in Japanese), Vol.69, No.687, A (2003), pp.1621-1627.

(11) JSME Standard S 001-1992, Standard Method of Test for Elastic-Plastic Fracture Toughness $\mathrm{J}_{\mathrm{IC}}$ (Supplement, 1st Edition), Japan Society of Mechanical Engineers, (1992).

(12) JSMS, Fracture Toughness Database (Metal Material), Edited by Fracture Mechanics Sub-Committee, The Society of Materials Science, Japan, (1995).

(13) Hutchinson, J.W., Singular Behavior at the End of a Tensile Crack in a Hardening Materials, J. Mech. Phys. Solids, Vol.16 (1968), pp.13-31.

(14) Rice, J.R. and Rosengren, G.F., Plane Strain Deformation near a Crack Tip in a Power-Law Hardening Material, J. Mech. Phys. Solids, Vol.16 (1968), pp.1-12.

(15) Tvergaard, V., On Localization in Ductile Materials Containing Spherical Voids, Int. J. Fracture, Vol.18 (1982), pp.237-252.

(16) Chu, C. and Needleman, A., Void Nucleation Effects in Biaxially Stretched Sheets, Trans. ASME, J. Enging. Mater. Tech., Vol.102 (1980), pp.249-256.

(17) Broek, D., The Role of Inclusions in Ductile Fracture and Fracture Toughness, Eng. Frac. Mech., Vol.5 (1973), pp.55-56.

(18) Kikuchi, M., Miyamoto, H., Otoyo, H. and Kuroda, M., Ductile Fracture of Aluminum Alloys (Observation of the Fracture Process by FRASTA and FEM Simulation), JSME Int. J., Ser. I, Vol.34, No.1 (1991), pp.9097. 